\title{
Interleukin 6 blockage-induced neutropenia in a patient with rheumatoid arthritis and resolved hepatitis $B$
}

\author{
Magdalena Chmielińska, Marzena Olesińska, Anna Felis-Giemza \\ Department of Connective Tissue Diseases, National Institute of Geriatrics, Rheumatology and Rehabilitation, Warsaw, Poland
}

\begin{abstract}
The authors present a case report of a 59-year-old woman with rheumatoid arthritis after documented recovery from hepatitis $\mathrm{C}(\mathrm{HCV})$ infection and with resolved HBV infection who has been undergoing successful tocilizumab treatment. The patient experienced moderate to severe neutropenia after consecutive tocilizumab administrations. However, no serious infections or HBV reactivation was recorded during that period.
\end{abstract}

Key words: hepatitis B, neutropenia, tocilizumab.

\section{Introduction}

Tocilizumab (TCZ) inhibits interleukin 6 (IL-6) signal transduction by competitively blocking its soluble receptor (sIL-6R) and membrane-expressed receptor present on the surface of many cells playing an important role in human immune defence [1]. The signalling block not only results in lower disease activity in rheumatoid arthritis (RA) but also might cause haematological changes such as neutropenia [2-8]. Interleukin 6 activity inhibition poses a risk of HBV reactivation, so proper monitoring of patients with resolved hepatitis $B$ is very important as antiviral treatment can be used at the right time.

The article describes a case of a woman suffering from rheumatoid arthritis (RA) with a history of hepatitis $B$ and C, who has been undergoing successful TCZ therapy.

\section{Case report}

A 59-year-old woman with a 9-year (2006) history of RA had poorly controlled disease despite treatment with synthetic disease-modifying anti-rheumatic drugs (DMARDs) including methotrexate (discontinued due to significant increase of liver enzymes alanine and aspartate transferase AST/ALT > 3 ULN), sulfasalazine and etanercept (lack of adequate response to treatment). None of these drugs fully controlled the disease activity.
Additionally, the patient did not receive chloroquine due to ophthalmological contraindications. Her Larsen's radiological grade was stage III. It was positive for rheumatoid factor (RF) and anti-cyclic citrullinated peptide antibodies (anti-CCP) were absent. In 2008 the patient was diagnosed with chronic HCV infection after abnormal liver function tests (LFT) and HCV RNA detection. The route of transmission was probably blood transfusion, which took place 25 years ago.

After three months of therapy with interferon $\alpha$ (INF- $\alpha$ ) and ribavirin the HCV RNA turned to negative and antiHCV treatment was completed after one year. Furthermore, a hepatologist recognized non-active hepatitis $B$ infection (surface HBs antigen and HBV DNA negative, anti-HBC antibody was present). For the first time the HBV infection was diagnosed and treated in 1979 but there were no records from that period.

Because of high RA activity (DAS28 $=6.6$ ) it was decided after consultation with a hepatologist to start tocilizumab therapy with a dose of $8 \mathrm{mg} / \mathrm{kg}$ monthly from November 2010. The hepatologist recommended regular testing of serum HBs antigen due to the risk of HBV reactivation during biological treatment.

All haematological parameters (haemoglobin, mean cell haemoglobin $[\mathrm{MCH}]$, mean corpuscular volume $[\mathrm{MCV}]$, red blood cell count $[\mathrm{RBC}]$, total white blood cell 
[WBC] count, platelet [PLT] count) and hepatic transaminase levels were in normal range before starting therapy. Liver ultrasonography revealed no abnormalities.

A significant clinical response to RA treatment was gained (DAS28 $=2.03$ ) in December 2010. There was observed a decrease in WBC level from $6820 / \mu$ to $3160 / \mu l$ after the first TCZ administration. Four times TCZ therapy was interrupted due to neutropenia, according to recommendations in Summary of Product Characteristics [9]. In the case of lowering the number of leukocytes the dose of TCZ was reduced to $4 \mathrm{mg} / \mathrm{kg}$. Moderate to severe neutropenia was recorded one day after a dose of TCZ (Table I). However, at the end of the 4-week dosing interval the neutrophil count usually returned to the normal level. Blood levels of folic acid, vitamin $B_{12}$, ferritin, and iron were in the normal range.

The patient was instructed to pay particular attention to avoid infections, especially after TCZ administration. From the beginning of tocilizumab therapy until now only once have urinary tract infection and symptoms of herpes labialis with a benign course been observed. LFT were performed every month and values remained in the normal range.
As well as HBV DNA measured after 2 years of TCZ therapy and HBsAg checked every month remained negative.

\section{Discussion}

The leucopenia phenomenon associated with TCZ administration was explained by Gibiansky and Frey. The sIL-6R-neutrophil count model is based on the assumption that neutrophil course during TCZ therapy is directly connected with the changes in unbound SIL-6R concentrations [1]. The fast effect of tocilizumab on neutrophil counts is related to the short neutrophil life span in circulation and quick IL-6 effect on neutrophil margination.

The authors measured neutrophil counts in two groups receiving $8 \mathrm{mg} / \mathrm{kg}$ and $4 \mathrm{mg} / \mathrm{kg}$ doses. Two weeks after TCZ administration they noted a decline in neutrophil counts, but they did not mention neutropenia. Presumably two weeks after the dose the majority of tocilizumab-sIL-6R complexes had already broken down and released sIL-6R receptors, which enabled IL-6 activity. According to the presented model it caused neutrophil

Table I. WBC and neutrophil count measured before and one day after tocilizumab administration. No increase in infection was the reason eventually we checked WBC and neutrophil count every 4 weeks (some data)

\begin{tabular}{|c|c|c|c|c|c|c|}
\hline \multirow[t]{2}{*}{ Date of administration } & \multicolumn{2}{|c|}{ Before TCZ } & \multirow[t]{2}{*}{ DAS28 } & \multirow{2}{*}{$\begin{array}{c}\text { TCZ } \\
{[\mathrm{mg} / \mathrm{kg}]}\end{array}$} & \multicolumn{2}{|c|}{1 day after TCZ } \\
\hline & $\begin{array}{c}\mathrm{WBC} / \mu \mathrm{l} \\
{[4000-10000]}\end{array}$ & $\begin{array}{c}\text { Neut/} / \mu \mathrm{l} \\
{[2500-6000]}\end{array}$ & & & $\begin{array}{c}\mathrm{WBC} / \mu \mathrm{l} \\
{[4000-10000]}\end{array}$ & $\begin{array}{c}\text { Neut} / \mu \mathrm{l} \\
{[2500-6000]}\end{array}$ \\
\hline 19.09.2011 & 6910 & 5530 & 5.51 & 8 & 1900 & 684 \\
\hline 28.10 .2011 & 3260 & 1450 & 0.28 & 4 & 1900 & 500 \\
\hline 07.12 .2011 & 6860 & 5380 & 5.53 & 8 & 1510 & 530 \\
\hline 11.01.2012 & 2660 & 1110 & 2.44 & 4 & 1880 & 600 \\
\hline$\ldots$ & $\ldots$ & $\ldots$ & $\ldots$ & $\ldots$ & $\ldots$ & $\ldots$ \\
\hline 10.05.2012 & 5460 & 3830 & 4.76 & 8 & 1670 & 420 \\
\hline 26.06 .2012 & 5780 & 4220 & 4.31 & 8 & 1310 & 310 \\
\hline$\ldots$ & $\ldots .$. & $\cdots$ & $\ldots$ & $\ldots$ & $\cdots$ & $\cdots$ \\
\hline 25.01.2013 & 4700 & 3130 & 4.02 & 4 & 1590 & 420 \\
\hline 8.03 .2013 & 6900 & 5250 & 6.63 & 8 & 1440 & 360 \\
\hline$\ldots .$. & $\ldots .$. & $\ldots .$. & $\cdots$ & $\cdots$ & $\cdots$ & $\ldots$ \\
\hline 24.11.2014 & 2460 & 1030 & 2.96 & 4 & $\ldots$ & $\ldots .$. \\
\hline 22.12.2014 & 3770 & 2210 & $\ldots$. & 8 & $\ldots$ & $\ldots$. \\
\hline$\ldots$ & $\cdots$ & $\ldots$ & $\ldots$ & $\cdots$ & $\ldots$ & $\ldots$ \\
\hline 16.10.2015 & 5250 & 4210 & 2.01 & 8 & $\ldots$ & $\ldots$ \\
\hline 19.11.2015 & 1900 & 960 & $\ldots$. & 4 & $\ldots$ & $\ldots$ \\
\hline
\end{tabular}


recovery, its rise and return to the baseline level (by the time of the next dose in the $4 \mathrm{mg} / \mathrm{kg}$ group and after the end of TCZ dosing in the $8 \mathrm{mg} / \mathrm{kg}$ group). The slower recovery after the $8 \mathrm{mg} / \mathrm{kg}$ dose than the $4 \mathrm{mg} / \mathrm{kg}$ dose is similar to our observations. The simulations which showed the direct relationship between increase in total tocilizumab concentration and total sIL-6R (bound and unbound with TCZ) and decline in free sIL-6R concentration and neutrophil count seem to clarify the following haematological changes.

Another hypothesis which may explain neutropenia occurrence after IL-6 blockage is slower neutrophil release from bone marrow or neutrophil accelerated apoptosis [8]. Wright et al. [8] investigated the influence of IL-6 and IL-6 blockage on neutrophils in vitro and in vivo. They not only observed no increase in neutrophil apoptosis but also found out that host defense associated with its function is not impaired after IL-6 blockage. This is consistent with available literature as it has not been reported that a reduction in neutrophil count during TCZ therapy is related to a significant increase in infection [3-6].

Nagamine et al. [2] measured complete blood count immediately before and after TCZ administration. They noted a significant decrease in WBC, neutrophil and lymphocyte levels. In two cases there was leucopenia with average $W B C<4000 / \mu l$ and its recovery after one week.

Similar observations in leucocyte number were made in the case described. It reached a value below 4000/ $\mu \mathrm{l}$ one day after a dose, and it took at least 3 weeks to return to the normal level.

As there was no increase in the infection incidence, we decided to check the WBC and neutrophil count only on the day of TCZ administration (which was every 4 weeks).

Nowadays we do not have a clear procedure how to monitor and treat patients with rheumatic disease and current or past hepatitis B infection. We may draw on recommendations from the European Association for the Study of the Liver (EASL) and current expert opinions in the literature $[10,11]$. It remains clear that screening for hepatitis B prior to biological therapy and periodical follow-up of liver enzymes and the activity of the virus (viraemia) in hepatitis-positive patients is crucial $[5,12]$. According to the guidelines from EASL, antiviral prophylaxis is recommended for patients with chronic hepatitis B. In the case of resolved hepatitis B, prophylaxis should be dependent on presence of HBV DNA and viral load [10]. Standards of medical care for patients with resolved hepatitis $B$ in Poland do not include HBV DNA monitoring. Only regular monitoring of $\mathrm{HBsAg}$ is recommended.

There are not enough data about risk of reactivation of HBV during tocilizumab therapy as patients screened positively for hepatitis $B$ were excluded from clinical trials [7]. However, it is clear that patients after HBV infection during chemotherapy or immunosuppressive treatment have increased risk of virus reactivation [10].

Japanese experience on RA patients with resolved hepatitis $B$ showed higher frequency of reactivation of HBV in the course of biological treatment (including $\mathrm{TCZ}$ ). Additionally there was no relationship between HBV reactivation and patients' age at presentation, RA duration, male gender, use of methotrexate (MTX) or cyclosporine (CS), dose of MTX and CS, levels of transaminases (ALT and AST), levels of immunoglobulin (IgG), neutrophil counts and lymphoid cell counts [13].

On the other hand, Nagashima and Minota encountered a case of a patient with 6.5-year-long tocilizumab therapy, who was found later to be a HBV carrier with a high HBV viral load. Interestingly, during all that time there were no adverse effects or HBV exacerbation reported [14].

Interleukin 6 has an important role in host defence and may contribute to HBV elimination [15]. It seems coherent that tocilizumab might have an influence on the course of chronic hepatitis, but there are still not enough data.

\section{Summary}

In the present case the decision about starting and continuing TCZ therapy seems to be appropriate. The patient gained low disease activity in a short time without reactivation of HBV infection or high frequency of other infections due to neutropenia. Tocilizumab was the better treatment to achieve complete control of disease activity. In the case described above we took into account all the risk of HBV reactivation, while the HCV infection was cured and there was no need to repeat the test for HCV RNA.

However, in the presence of risk factors such as history of hepatitis virus infections, safety of TCZ treatment should be confirmed in randomized control trials. Also the emergence of a separate recommendation for patients with previous history or infection with hepatitis B or $\mathrm{C}$ and treated with TCZ is expected.

The authors declare no conflict of interest.

\section{References}

1. Gibiansky L, Frey N. Linking interleukin-6 receptor blockade with tocilizumab and its hematological effects using a modeling approach. J Pharmacokinet Pharmacodyn 2012; 39: 5-16.

2. Nagamine R, Chen W, Hara T, et al. Immediate reduction of white blood cell count after tocilizumab administration was observed in some cases. Mod Rheumatol 2009; 19: 348-350. 
3. Nisar MK, Östör AJK. The role of tocilizumab monotherapy In the management of rheumatoid arthiritis: a review. Int J Clin Rheumatol 2012; 7: 9-19.

4. Nishimoto N, Miyasaka N, Yamamoto K, et al. Long-term safety and efficacy of tocilizumab, an anty-interleukin- 6 receptor monoclonal antibody, in monotherapy, in patients with rheumatoid arthritis (the STREAM study): evidence of safety and efficacy in a 5-year extension study. Ann Rheum Dis 2009; 68: 1580-1584.

5. Navarro-Millan I, Singh JA, Curtis JR. Systemic review of tocilizumab for rheumatoid arthritis: a new biologic agent targeting the interleukin-6 receptor. Clin Ther 2012; 34: 788-800.

6. Jones G, Sebba A, Gu J, et al. Comparison of tocilizumab monotherapy versus methotrexate monotherapy in patients with moderate to severe rheumatoid arthritis: the AMBITION study. Ann Rheum Dis 2010; 69: 88-96.

7. Rubbert-Roth A. Assessing the safety of biologic agents in patients with rheumatoid arthritis. Rheumatology (Oxford) 2012; 51: v38-v47.

8. Wright H, Cross A, Edwards S, et al. Effects of IL- 6 and IL-6 blockade on neutrophil function in vitro and in vivo. Rheumatology 2014; 53: 1321-1331.

9. European Medicines Agency. Roactemra: EPAR - Product Information. Annex I. Summary of product characteristics.

10. European Association for the Study of the Liver. EASL. Clinical Practice Guidelines: management of chronic hepatitis B virus infection. J Hepatol 2012; 57: 167-185.

11. Vassilopoulos D, Calabrese LH. Management of rheumatic disease with comorbid HBV or HCV infection. Nat Rev Rheumatol 2012; 8: 348-357.

12. Keyser FD. Choice of biologic therapy for patients with rheumatoid arthritis: the infection perspective. Curr Rheumatol Rev 2011; 7: 77-87.

13. Urata $Y$, Uesato $R$, Tanaka $D$, et al. Prevalence of reactivation of hepatitis B virus replication in rheumatoid arthritis patients. Mod Rheumatol 2011; 21: 16-23.

14. Nagashima T, Minota S. Long-term tocilizumab therapy in a patient with rheumatoid arthritis and chronic hepatitis B. Rheumatology 2008; 47: 1838-1840.

15. Kakumu S, Shinagawa T, Ishikawa T, et al. Serum interleukin 6 levels in patients with chronic hepatitis B. Am J Gastroenterol 1991; 86: 1804-1808. 